\title{
Flexural vibration of pyrocomposite solid cylinder
}

\author{
Nelson V. K. ${ }^{a}$ And Karthikeyan S., b \\ a Department of Mathematics, Government College of Engineering, Bargur - 635 104, India) \\ ${ }^{\mathrm{b}}$ Department of Mathematics, Sona College of Technology, Salem - 636 005, India \\ *To whom correspondence should be addressed. E-mail: rishabkarthik1@gmail.com
}

Received 10 January 2008

http://dx.doi.org/10.11113/mjfas.v4n1.36

\begin{abstract}
Flexural vibration of an infinite Pyrocomposite circular cylinder made of inner solid and outer hollow pyroelectric layer belonging to $6 \mathrm{~mm}$-class bonded together by a Linear Elastic Material with Voids (LEMV) is studied. The exact frequency equation is obtained for the traction free outer surface with continuity conditions at the interfaces. Numerical results in the form of data and dispersion curves for the first and second mode of the flexural vibration of the cylinder ceramic - 1 / Adhesive / ceramic - 2 by taking the adherents as $\mathrm{BaTio}_{3}$ and the adhesive layer as an existing Carbon Fibre Reinforced Polymer (CFRP) or as a hypothetical LEMV layer with and without voids are compared with a pyroelectric solid cylinder. The damping is analyzed through the imaginary part of the complex frequencies.
\end{abstract}

| Flexural vibration | pyrocomposite | solid cylinders | LEMV | CFRP |

\section{INTRODUCTION}

The applications of pyroelectric materials and pyroelectric ceramic/polymer composite materials are many and [1] - [10] are a few in particular. Dispersion characteristics of wave propagation in pyoelectric plate and cylinder have been studied by Paul and Raman [11]-[13]. Paul and Nelson [14] have extended the study of Vasudeva and Govinda Rao [15]-[16] on the influence of distributed voids in the interfacial LEMV adhesive zones of the isotropic Sandwich plate to the flexural vibration of Piezo composite hollow cylinder. A continuum theory of LEMV with distinct properties has been developed by Cowin and Nunziato [17]. In layered composites pores or voids are found in the interface region and it is known to affect the estimation of physical and mechanical properties of the composites [18]. Voorhees and Green [19] have studied the mechanical behavior of sandwich composites made of thin porous core and denser face materials. Damage detection and vibration control of a new smart board designed by mounting piezoelectric fibers with metal cores on the surface of a CFRP composite was studied by Takagikiyoshi [20].

In the present analysis flexural vibration of pyrocomposite circular solid cylinder of crystal class 6mm with LEMV/CFRP as a bonding layer is considered. The frequency equation for flexural vibration of solid cylinder has been derived for traction free shorted outer surface with interface continuity conditions on both sides of the LEMV layer. Numerical work is carried out and the dispersion curves for the flexural vibration of the 
pyrocomposite solid cylinder with middle core LEMV/CFRP are compared with that of pyroelectric solid cylinder.

\section{GOVERNING EQUATIONS}

The equations governing elastic, electric and thermal behavior are given by Mindlin [21] - [22]

$$
\begin{array}{ll}
T^{l}{ }_{i j, j}=\rho^{l} u^{l}{ }_{i, t t}, & T^{l}{ }_{i j}=C^{l}{ }_{i j k l} S^{l}{ }_{k l}-e^{l}{ }_{k i j} E^{l}{ }_{k}-\lambda^{l}{ }_{i j} \theta^{l}, \\
D^{l}{ }_{i, i}=0, & \text { and } \quad D^{l}{ }_{j}=e^{l}{ }_{j k l} S^{l}{ }_{k l}+\varepsilon^{l}{ }_{j k} E^{l}{ }_{k}+p^{l}{ }_{j} \theta^{l}, \\
k^{l}{ }_{i j} \theta^{l}{ }_{i j}=\theta^{l}{ }_{0} \sigma^{l}{ }_{, t} & \sigma^{l}=\lambda^{l}{ }_{k l} S^{l}{ }_{k l}+p^{l}{ }_{k} E^{l}{ }_{k}+\alpha^{l} \theta^{l} \quad \text { and } \alpha^{l}=\rho^{l} C^{l}{ }_{v} \theta_{0}{ }^{-1}{ }^{l}
\end{array}
$$

Where, $T^{l}{ }_{i j}, S_{k l}{ }^{l}, D^{l}{ }_{j}, E_{k}{ }^{l}, \sigma^{l}$ and $\theta^{l}$ are stresses, strains, electric displacements, electric fields, entropy and temperature. Here, $C_{v}^{l}$ is the specific heat capacity, $\theta_{0}$ is the reference temperature, and $\rho^{l}$ is the density. And $C^{l}{ }_{i j}, e^{l}{ }_{m n}, \varepsilon^{l}{ }_{k l}, \beta^{l}{ }_{j}$ and $p^{l}{ }_{m}$ are elastic, piezoelectric, dielectric, thermal stress coefficients and pyroelectric constants respectively. The comma followed by an independent variable denotes partial differentiation of that coefficient with respect to that independent variable and $E_{i}^{l}=-\phi^{l},{ }_{i}$. The superscript $(l=1,2)$ is to denote the constants and variables of inner and outer pyroelectric materials of hexagonal (class 6 $\mathrm{mm})$.

For crystal class $6 \mathrm{~mm}$, the material constants are

$$
\begin{aligned}
& C^{l}=\left[\begin{array}{cccccc}
C_{11}^{l} & C_{12}^{l} & C_{13}^{l} & 0 & 0 & 0 \\
C_{12}^{l} & C_{11}^{l} & C^{l}{ }_{23} & 0 & 0 & 0 \\
C_{13}^{l} & C_{23}^{l} & C_{33}^{l} & 0 & 0 & 0 \\
0 & 0 & 0 & C^{l}{ }_{44} & 0 & 0 \\
0 & 0 & 0 & 0 & C^{l}{ }_{44} & 0 \\
0 & 0 & 0 & 0 & 0 & C_{66}^{l}
\end{array}\right], \beta^{l}=\left[\begin{array}{c}
\beta_{1}^{l} \\
\beta^{l}{ }_{2} \\
\beta^{l}{ }_{3} \\
0 \\
0 \\
0
\end{array}\right], p^{l^{T}}=\left[\begin{array}{c}
0 \\
0 \\
p^{l}{ }_{3}
\end{array}\right] \\
& e^{l}=\left[\begin{array}{cccccc}
0 & 0 & 0 & e^{l}{ }_{14} & e^{l}{ }_{15} & 0 \\
0 & 0 & 0 & e^{l}{ }_{15} & -e^{l}{ }_{14} & 0 \\
e_{31}^{l} & e^{l}{ }_{31} & e_{33}^{l} & 0 & 0 & 0
\end{array}\right], \varepsilon^{l}=\left[\begin{array}{ccc}
\varepsilon^{l}{ }_{11} & 0 & 0 \\
0 & \varepsilon^{l}{ }_{11} & 0 \\
0 & 0 & \varepsilon^{l}{ }_{33}
\end{array}\right]
\end{aligned}
$$

Where, $C_{66}^{l}=\left(\frac{C_{11}^{l}-C_{12}^{l}}{2}\right)$ The stress components $T^{l}{ }_{i j}$, electric displacements $D_{m}$ and the entropy $\sigma$ satisfy the following equations for flexural vibration of hexagonal symmetry 


$$
\begin{aligned}
& T_{r r, r}^{l}+r^{-1} T_{r \theta, r}^{l}+T_{r z, z}^{l}+r^{-1}\left(T_{r r}^{l}-T_{\theta \theta}^{l}\right)=\rho^{l} u^{l}{ }_{t t}, \\
& T_{\theta z, z}^{l}+r^{-1} T_{\theta \theta, \theta}^{l}+T_{r \theta, \theta}^{l}+2 r^{-1} T_{r \theta}^{l}=\rho^{l} v_{, t t}^{l} \\
& T_{z z, z}^{l}+T_{\theta z, \theta}^{l}+T_{r z, r}^{l}+r^{-1} T_{r z}^{l}=\rho^{l} w^{l},_{t t}, \\
& r^{-1}\left[r D_{r, r}^{l}+D_{r}^{l}+D_{\theta, \theta}^{l}\right]+D_{z, z}^{l}=0, \\
& k^{l}{ }_{11} \nabla^{2} T^{l}+k^{l}{ }_{33} T^{l}{ }_{z z}=T^{l}{ }_{0} \sigma^{l}{ }_{t},
\end{aligned}
$$

where ,

$$
\bar{\nabla}^{2}=\frac{\partial^{2}}{\partial r^{2}}+r^{-1} \frac{\partial}{\partial r}+r^{-2} \frac{\partial^{2}}{\partial \theta^{2}}
$$

The equations of flexural motion, Gauss's equation and the entropy equation in cylindrical polar coordinates r, $\theta$, $\mathrm{z}$ for class 6 are

$$
\begin{aligned}
& c_{11}^{l}\left[u^{l}, r r+r^{-1} u^{l},{ }_{r}+r^{-2} u^{l}\right]+c_{66}^{l} r^{-2} u_{, \theta \theta}+c^{l}{ }_{44} u^{l}{ }{ }_{z z}+\left(c_{66}^{l}+c_{12}^{l}\right) r^{-1} v_{, r}-\left(c_{66}^{l}+c_{11}^{l}\right) r^{-2} v_{, \theta} \\
& +\left(c^{l}{ }_{44}+c^{l}{ }_{13}\right) w^{l},{ }_{r z}+\left(e^{l}{ }_{31}+e^{l}{ }_{15}\right) \phi^{l},{ }_{r z}-e_{14} r^{-1} \phi_{, \theta z}^{l}-\beta^{l}{ }_{1} T^{l},{ }_{r}=\rho^{l} u^{l},{ }_{t t}, \\
& \left(c_{66}^{l}+c_{11}^{l}\right) r^{-1} u^{l},_{r \theta}+\left(c_{66}^{l}+c_{11}^{l}\right) r^{-2} u,{ }_{\theta}+c_{66}^{l}\left(v,{ }_{r r}+r^{-1} v,{ }_{r}-r^{-2} v^{l}\right)+c_{11}^{l} r^{-2} v^{l},{ }_{\theta \theta}+c_{44}^{l} v,{ }_{z z} \\
& +\left(c_{44}^{l}+c_{13}^{l}\right) r^{-1} w^{l},_{\theta z}+e_{14}^{l} \phi^{l},_{r z}+\left(e_{31}^{l}+e^{l}{ }_{15}\right) r^{-1} \phi_{, \theta z}^{l}-r^{-1} \beta_{1}^{l} T_{, \theta}^{l}=\rho^{l} v_{, t t}^{l} \\
& \left(c^{l}{ }_{44}+c^{l}{ }_{13}\right)\left[u^{l}{ }_{, r z}+r^{-1}\left(u^{l},{ }_{z}+v^{l}, \theta z\right)\right]+c^{l}{ }_{44}\left(w^{l},{ }_{r r}+r^{-1} w^{l},{ }_{r}+r^{-2} w_{, \theta \theta}^{l}\right)+c^{l}{ }_{33} w^{l},{ }_{z z}+ \\
& e^{l}{ }_{15}\left(\phi^{l},{ }_{r r}+r^{-1} \phi^{l},{ }_{r}+r^{-2} \phi_{, \theta \theta}^{l}\right)+e_{33} \phi^{l},{ }_{z z}-\beta^{l}{ }_{3} T^{l},{ }_{z}=\rho^{l} w^{l},{ }_{t t}, \\
& \varepsilon_{11}^{l}\left(\phi^{l},_{r r}+r^{-1} \phi^{l},{ }_{r}+r^{-2} \phi_{, \theta \theta}^{l}\right)+\varepsilon_{33}^{l} \phi^{l},_{z z}-\left(e^{l}{ }_{31}+e^{l}{ }_{15}\right)\left[u^{l},_{r z}+r^{-1}\left(u_{, z}^{l}+v_{, \theta z}^{l}\right)\right] \\
& +e^{l}{ }_{14}\left[r^{-1}\left(u_{, \theta z}-v_{, z}\right)-v_{, r z}\right]-e^{l}{ }_{15}\left(w^{l},{ }_{r r}+r^{-1} w^{l},{ }_{r}+r^{-2} w^{l}, \theta \theta\right)-e_{33} w^{l},_{z z}-p^{l}{ }_{3} T^{l},{ }_{z}=0 \text {, } \\
& k_{11}^{l}\left[T^{l},_{r r}+r^{-1} T^{l},_{r}+r^{-2} T_{, \theta \theta}^{l}\right]+k_{33}^{l} T^{l},_{z z}-T_{0}^{l} d^{l} T^{l},_{t} \\
& =T_{0}^{l}\left[\beta_{1}^{l}\left(u^{l},_{r t}+r^{-1} u^{l},{ }_{t}+r^{-1} v_{, \theta t}\right)+\beta^{l}{ }_{3} w^{l},_{z t}-p^{l}{ }_{3} \phi^{l},_{z t}\right] \text {, }
\end{aligned}
$$

Where $k_{i i}$ is heat conduction coefficient, $d=\frac{\rho C_{v}}{T_{0}}, \mathrm{u}, \mathrm{v}$ and $\mathrm{w}$ are the displacements along $\mathrm{r}, \theta, \mathrm{z}$ direction, $\phi$ is the electric potential, $\rho$ is the mass density and $t$ is the time. The solutions of Eqn. (3) is considered in the form

$$
\begin{aligned}
& u^{l}(r, \theta, z, t)=\left(u^{l},{ }_{r}+r^{-1} v_{, \theta}^{l}\right) \exp \{i(k z+p t)\}, \\
& v^{l}(r, \theta, z, t)=\left(r^{-1} u_{, \theta}^{l}-v_{, r}^{l}\right) \exp \{i(k z+p t)\},
\end{aligned}
$$




$$
\begin{aligned}
& w^{l}(r, \theta, z, t)=i\left(\frac{w^{l}}{h}\right) \exp \{i(k z+p t)\}, \\
& \phi^{l}(r, \theta, z, t)=i\left(\frac{C^{l}{ }_{44}}{e^{l}}\right)\left(\frac{\phi^{l}}{h}\right) \exp \{i(k z+p t)\}, \\
& T^{l}(r, \theta, z, t)=\left(\frac{C^{l}{ }_{44}}{\beta^{l}}\right)\left(\frac{T^{l}}{h^{2}}\right) \exp \{i(k z+p t)\}
\end{aligned}
$$

Where, $\mathrm{k}$ is the wave number, $\mathrm{p}$ is the angular frequency and $i=\sqrt{-1}$. We introduce the non-dimensional quantities $\mathrm{x}$ and $\varepsilon$ such that $x=\left(\frac{r}{h}\right), \varepsilon=k h$ and $\mathrm{h}=\mathrm{h}_{3}-\mathrm{h}_{0}\left(\mathrm{~h}_{0}, \mathrm{~h}_{3}\right.$ are inner and outer radius of the cylinders) thickness of the composite solid cylinder.

Using the above solution, Eqn (3) can be rewritten as

$$
\begin{array}{cccc|}
\bar{C}_{11}^{l} \nabla^{2}+A_{1} & -A_{2} & A_{3} & -A_{4} \\
A_{2} \nabla^{2} & \bar{c}_{44}{ }^{l} \nabla^{2}+A_{5} & \left(\bar{e}_{15}^{l} \nabla^{2}+A_{6}\right) \bar{c}_{44}{ }^{l} & A_{7} \\
A_{3} \nabla^{2} & \bar{e}_{15}^{l} \nabla^{2}+A_{6} & -\left(K_{33}^{-2} \nabla^{2}+A_{8}\right) & -A_{9} \\
A_{4} \nabla^{2} & A_{7} & -A_{9} & i \bar{K}_{1}^{l} \nabla^{2}+A_{10}
\end{array} \mid\left(u^{l}, w^{l}, \phi^{l}, T^{l}\right)=0,
$$

$$
\left[\bar{C}_{66}^{l} \nabla^{2}+A_{1}\right] v^{l}=0
$$

Where

$$
\begin{aligned}
& \nabla^{2}=\frac{\partial^{2}}{\partial x^{2}}+\left(\frac{1}{x}\right) \frac{\partial}{\partial x} \\
& A_{1}=\varepsilon^{2}-(c h)^{2} \\
& A_{2}=\varepsilon\left(1+\bar{C}_{13}^{l}\right) \\
& A_{3}=\varepsilon\left(\bar{e}_{31}^{l}+\bar{e}_{15}^{l}\right) \\
& A_{4}=\bar{\beta} \\
& A_{5}=\varepsilon^{2} \epsilon_{33}^{l}-(c h)^{2} \\
& A_{6}=\varepsilon^{2} \\
& A_{7}=\varepsilon \\
& A_{8}=K_{33}^{2} \varepsilon^{2} \\
& A_{9}=p^{l} \varepsilon \\
& A_{10}=i K_{3} \varepsilon^{2}-d
\end{aligned}
$$


and

$$
\begin{aligned}
& \bar{C}_{i j}^{l}=\frac{C^{l}{ }_{i j}}{C^{1}{ }_{44}}, \quad \bar{e}_{i j}=\frac{e^{l}{ }_{i j}}{e^{l}{ }_{33}}, \quad \bar{\beta}=\frac{\beta^{l}}{\beta^{l}{ }_{3}}, \quad \bar{d}^{l}=\frac{\rho^{l} C^{l}{ }_{44} C^{l}{ }_{v}}{\beta^{l^{2}} \theta_{0}}, \\
& \bar{K}_{i j}^{2}=\frac{\varepsilon^{l}{ }_{i j} C^{l}{ }_{44}}{e^{l^{2}}}, \quad \bar{K}_{i j}=\frac{\left(\rho^{l} C^{l}{ }_{44}\right)^{1 / 2} K^{l}{ }_{i j}}{\beta^{l^{2}} h \theta^{l}{ }_{0}(c h)}, \bar{p}=\frac{p_{3} C_{44}}{e_{33} \beta_{3}}, \bar{k}_{i}=\frac{k_{i}}{c h}
\end{aligned}
$$

The solutions of the Eqn (5) are taken as

$$
\begin{aligned}
& u^{l}=\sum_{j=0}^{4}\left[A_{j}^{l} J_{n}\left(\alpha_{j}^{l} x\right)+B_{j}^{l} Y_{n}\left(\alpha_{j}^{l} x\right)\right] \cos n \theta \\
& w^{l}=\sum_{j=0}^{4}\left[A_{j}^{l} J_{n}\left(\alpha_{j}^{l} x\right)+B_{j}^{l} Y_{n}\left(\alpha_{j}^{l} x\right)\right] d_{j}^{l} \cos n \theta \\
& \phi^{l}=\sum_{j=0}^{4}\left[A_{j}^{l} J_{n}\left(\alpha_{j}^{l} x\right)+B_{j}^{l} Y_{n}\left(\alpha_{j}^{l} x\right)\right] e_{j}^{l} \cos n \theta \\
& T^{l}=\sum_{j=0}^{4}\left[A_{j}^{l} J_{n}\left(\alpha_{j}^{l} x\right)+B_{j}^{l} Y_{n}\left(\alpha_{j}^{l} x\right)\right] h_{j}^{l} \cos n \theta
\end{aligned}
$$

And $\left(\alpha_{j}^{l} x\right)^{2}$ are the four roots of the Eqn (5) when replacing $\nabla^{2}=-\left(\alpha_{j}^{l} x\right)^{2}$.

The constants $d_{j}^{l}, e_{j}^{l}$ and $h_{j}^{l}$ can be evaluated using the following relations:

$$
\begin{aligned}
& \left(\bar{C}_{11}^{l} \nabla^{2}+A_{1}\right)-A_{2} d^{l}{ }_{j}+A_{3} e^{l}{ }_{j}-A_{4} h^{l}{ }_{j}=0, \\
& A_{2} \nabla^{2}+\left(\bar{c}_{44}{ }^{l} \nabla^{2}+A_{5}\right) d^{l}{ }_{j}+\left(\bar{e}_{15}^{l} \nabla^{2}+A_{6}\right) \bar{c}_{44}{ }^{l} e^{l}{ }_{j}-A_{7} h^{l}{ }_{j}=0, \\
& A_{3} \nabla^{2}+\left(\bar{e}_{15}^{l} \nabla^{2}+A_{6}\right) d^{l}{ }_{j}-\left(K_{33}^{-2} \nabla^{2}+A_{8}\right) e^{l}{ }_{j}-A_{9} h^{l}{ }_{j}=0, \\
& A_{4} \nabla^{2}+A_{7} d^{l}{ }_{j}-A_{9} e^{l}{ }_{j}+\left(i \bar{K}_{1}{ }^{l} \nabla^{2}+A_{10}\right) h^{l}{ }_{j}=0 .
\end{aligned}
$$

Also the solution of Eqn. (6) is $v^{l}=\left[A_{5}^{l} J_{n}\left(\alpha_{5}^{l} x\right)+B_{5}^{l} y_{n}\left(\alpha_{5}^{l} x\right)\right] \sin n \theta$, Where $\left(\alpha_{5}^{l} x\right)^{2}=\frac{A_{1}}{\bar{C}_{66}^{l}}$ 


$$
\begin{aligned}
& \rho \ddot{u}_{i}=\mu u_{i, j j}+(\lambda+\mu) u_{j, j i}+\beta \psi,_{i}, \\
& \rho k \ddot{\psi}=\alpha \psi_{, i i}-\omega \dot{\psi}-\xi \psi-\beta u_{k, k}
\end{aligned}
$$

Where $u, v, w$ are displacements in $\mathrm{r}, \theta$ and $\mathrm{z}$ directions $\alpha, \beta, \xi, \omega$ and $k$ (equilibrated inertia) are material constants characterizing the core of LEMV, $\rho$ is the density and $\lambda, \mu$ are the Lame's constants and $\psi$ is the new kinematical variable associated with a material with voids comes into contact with another material without voids.

The solution for Eqn. (9) is taken as

$u(r, \theta, z, t)=\left(u,{ }_{r}+r^{-1} v_{, \theta}\right) \exp \{i(k z+p t)\}$

$v(r, \theta, z, t)=\left(r^{-1} u_{, \theta}-v, r\right) \exp \{i(k z+p t)\}$,

$w(r, \theta, z, t)=i\left(\frac{w}{h}\right) \exp \{i(k z+p t)\}$,

$\psi(r, \theta, z, t)=\left(\frac{\psi}{h^{2}}\right) \exp \{i(k z+p t)\}$,

Substituting Eqn. (10) in Eqn (9) and using the dimensionless variables $\mathrm{x}$ and $\varepsilon$, the Eqn. (10) becomes

$$
\begin{aligned}
& (\bar{\lambda}+2 \bar{\mu}) \nabla^{2}+B_{1} \quad-B_{2} \quad B_{3} \\
& \begin{array}{ccc}
B_{2} \nabla^{2} & \bar{\mu} \nabla^{2}+B_{4} & B_{5}
\end{array}(u, w, \psi)=0 \\
& -B_{3} \nabla^{2} \\
& B_{5} \quad \bar{\alpha} \nabla^{2}+B_{6} \\
& {\left[\bar{\mu} \nabla^{2}+B_{1}\right] v=0 \text {. }}
\end{aligned}
$$

Where

$$
\begin{aligned}
& \nabla^{2}=\frac{\partial^{2}}{\partial x^{2}}+\left(\frac{1}{x}\right) \frac{\partial}{\partial x} \\
& B_{1}=\left(\frac{\rho}{\rho^{1}}\right)(c h)^{2}-\bar{\mu} \varepsilon^{2}, \\
& B_{2}=(\bar{\lambda}+\bar{\mu}) \varepsilon, \\
& B_{3}=\bar{\beta}, \\
& B_{4}=\left(\frac{\rho}{\rho^{1}}\right)(c h)^{2}-(\bar{\lambda}+2 \bar{\mu}) \varepsilon^{2}, \\
& B_{5}=\bar{\beta} \varepsilon, \\
& B_{6}=\left(\frac{\rho}{\rho^{1}}\right)(c h)^{2} \bar{k}-\bar{\alpha} \varepsilon^{2}-i \bar{\omega}(c h)-\bar{\xi},
\end{aligned}
$$


and

$$
\begin{gathered}
\bar{\lambda}=\frac{\lambda}{C_{44}^{1}}, \bar{\mu}=\frac{\mu}{C_{44}^{1}}, \bar{\alpha}=\frac{\alpha}{h^{2} C_{44}^{1}}, \bar{\beta}=\frac{\beta}{C^{1}{ }_{44}}, \\
\bar{\xi}=\frac{\xi}{C^{1}{ }_{44}}, \bar{\omega}=\left(\frac{\omega}{h}\right)\left(C^{1}{ }_{44} \rho\right)^{-\frac{1}{2}}, \bar{K}=\frac{k}{h^{2}}
\end{gathered}
$$

The solutions of the Eqn. (11) are taken as

$u=\sum_{j=0}^{3}\left[A_{j} J_{0}\left(\alpha_{j} x\right)+B_{j} y_{0}\left(\alpha_{j} x\right)\right] \cos n \theta$

$w=\sum_{j=0}^{3}\left[A_{j} J_{0}\left(\alpha_{j} x\right)+B_{j} y_{0}\left(\alpha_{j} x\right)\right] d_{j} \cos n \theta$

$\psi=\sum_{j=0}^{3}\left[A_{j} J_{0}\left(\alpha_{j} x\right)+B_{j} y_{0}\left(\alpha_{j} x\right)\right] e_{j} \cos n \theta$

$\left(\alpha_{j} x\right)^{2}$ are the three roots of the equation (11) when replacing $\nabla^{2}=-\left(\alpha_{j} x\right)^{2}$. The constants $d_{j}$ and $e_{j}$ can be evaluated using the following relations:

$B_{2} \nabla^{2}+\left(\bar{\mu} \nabla^{2}+B_{4}\right) d_{j}+B_{5} e_{j}=0$,

$-B_{3} \nabla^{2}+\left(B_{5}\right) d_{j}+\left(\alpha \nabla^{2}+B_{6}\right) e_{j}=0$

The solution of Eqn. (12) is

$v=\left[A_{4} J_{n}\left(\alpha_{4} x\right)+B_{4} y_{n}\left(\alpha_{4} x\right)\right] \sin n \theta$,

Where $\left(\alpha_{4} x\right)^{2}=\frac{B_{1}}{\bar{\mu}}$

The governing equation for CFRP core material can be deduced from Eqn. (9) by taking the void volume fraction $\psi=0$, and the Lame's constants as $\lambda=c_{12}, \mu=\frac{\left(c_{11}-c_{12}\right)}{2}$

\section{FREQUENCY EQUATIONS}

The frequency equation has been derived by using the following boundary and interface conditions

(i) Since the outer surface are traction free and coated with electrodes which is shorted, the boundary conditions become

$T_{r r}^{l}=T_{r \theta}^{l}=T_{r z}^{l}=\phi^{l}=T^{l}{ }_{r}=0$ at $r=x_{3}$ with $l=2$

(ii) On the interfaces (inner and middle, outer and middle), the continuity conditions are

At $r=x_{2}, x_{1}$

$T_{r r}^{l}=T_{r r} ; T_{r \theta}^{l}=T_{r \theta}, T_{r z}^{l}=T_{r z} ; u^{l}=u ; v^{l}=v ; w^{l}=w$

$\phi^{l}=0, T^{l}, r=0 \quad$ (At non-pyroelectric core material) and

$\psi_{, r}=0$ due to void volume fraction field with $l=1,2$ 
The interface condition $\psi_{, r}=0$ on the void volume fraction field $\psi$ is suggested by Atkin et al [24]. (When a material with voids comes into contact with another material without voids). The frequency equation is obtained as $23 \times 23$ determinantal equation, on substituting the solutions in the boundary- interface conditions. It is written in symbolic form as

$$
|E(i, j)|=0,(i, j=1,2,3, \ldots, 23)
$$

The non-zero elements at $x=x_{1}$ by varying $\mathrm{j}$ from 1 to 4 are

$$
\begin{aligned}
& E(1, j)=2 \bar{c}_{66}{ }^{1}\left(\frac{\alpha_{j}^{1}}{x_{1}}\right) J_{1}\left(\alpha_{j}^{1} x_{1}\right)-\left[\bar{c}_{11}\left(\alpha_{j}^{1}\right)^{2}+\bar{c}_{13}^{1} \varepsilon d_{j}^{1}+\bar{e}_{31}^{1} \varepsilon e_{j}^{1}+\bar{\beta} h_{j}\right] J_{0}\left(\alpha_{j}^{1} x_{1}\right) \\
& E(1,5)=-2 n \bar{c}_{66}^{1}\left(\frac{\alpha_{4}^{1}}{x_{0}}\right) J_{1}\left(\alpha_{5}^{1} x_{0}\right)+\left[\frac{2 n(n-1) \bar{c}_{66}^{1}}{\left.x_{0}\right)^{2}}\right] J_{0}\left(\alpha_{5}^{1} x_{0}\right) \\
& E(1, j+5)=-\left[\begin{array}{l}
2 \bar{\mu}\left(\frac{\alpha_{j}}{x_{1}}\right) J_{1}\left(\alpha_{j} x_{1}\right) \\
\left.+\left\{-(\bar{\lambda}+2 \bar{\mu})\left(\alpha_{j}\right)^{2}+\bar{\beta} e_{j}-\bar{\lambda} \varepsilon d_{j}+\frac{2 n(n-1) \bar{\mu}}{x_{0}^{2}}\right\} J_{0}\left(\alpha_{j} x_{1}\right)\right] \\
E(1,9)=\left[2 n \bar{\mu}\left(\frac{\alpha_{4}}{x_{1}}\right) J_{1}\left(\alpha_{4} x_{1}\right)-\left(\frac{(n-1)}{x_{0}^{2}}\right) J_{0}\left(\alpha_{4} x_{1}\right)\right] \\
E(2, j)=2 n \bar{c}_{66}^{1}\left\{\left(\frac{\alpha_{j}^{1}}{x_{1}}\right) J_{1}\left(\alpha_{j}^{1} x_{1}\right)-\left[\frac{(n-1)}{\left(x_{1}\right)^{2}}\right] J_{0}\left(\alpha_{j}^{1} x_{1}\right)\right\} \\
E(2,5)=\bar{c}_{66}^{1}\left\{-2\left(\frac{\alpha_{5}^{1}}{x_{1}}\right) J_{1}\left(\alpha_{5}^{1} x_{1}\right)+\left[\left(\alpha_{5}^{1} x_{1}\right)^{2}-\frac{2 n(n-1)}{\left(x_{1}\right)^{2}}\right] J_{0}\left(\alpha_{5}^{1} x_{1}\right)\right\} \\
E(2, j+5)=-2 n \bar{\mu}\left[\left(\frac{\alpha_{j}}{x_{1}}\right) J_{1}\left(\alpha_{j} x_{1}\right)-\left[\frac{(n-1)}{\left(x_{1}\right)^{2}}\right] J_{0}\left(\alpha_{j} x_{1}\right)\right] \\
E(2,9)=2 \bar{\mu}\left[\left(\frac{\alpha_{4}}{x_{1}}\right) J_{1}\left(\alpha_{4} x_{1}\right)+\left(\frac{n(n-1)}{\left(x_{1}\right)^{2}}-\left(\frac{\left(\alpha_{4}\right)^{2}}{2}\right)\right) J_{0}\left(\alpha_{4} x_{1}\right)\right] \\
E(3, j)=-\left(\varepsilon+d_{j}^{1}+\bar{e}_{15}^{1} e_{j}^{1}\right)\left[\left(\alpha_{j}^{1}\right) J_{1}\left(\alpha_{j}^{1} x_{1}\right)-\left(\frac{n}{x_{1}}\right) J_{0}\left(\alpha_{j}^{1} x_{1}\right)\right]
\end{array}\right. \\
& E
\end{aligned}
$$




$$
\begin{aligned}
& E(3,5)=\left(\frac{n}{x_{1}}\right) \varepsilon J_{0}\left(\alpha_{5}^{1} x_{1}\right) \\
& E(3, j+5)=-\bar{\mu}\left(\varepsilon+d_{j}\right)\left[-\alpha_{j} J_{1}\left(\alpha_{j} x_{1}\right)+\left(\frac{n}{x_{1}}\right) J_{0}\left(\alpha_{j} x_{1}\right)\right] \\
& E(3,9)=-\bar{\mu}\left(\frac{n}{x_{1}}\right) \varepsilon J_{0}\left(\alpha_{4} x_{1}\right) \\
& E(4, j)=-\alpha_{j}^{1} J_{1}\left(\alpha_{j}^{1} x_{1}\right)+\left(\frac{n}{x_{1}}\right) J_{0}\left(\alpha_{j}^{1} x_{1}\right) \\
& E(4,5)=\left(\frac{n}{x_{1}}\right) J_{0}\left(\alpha_{5}^{1} x_{1}\right) \\
& E(4, j+5)=\alpha_{j} J_{1}\left(\alpha_{j} x_{1}\right)-\left(\frac{n}{x_{1}}\right) J_{0}\left(\alpha_{j} x_{1}\right) \\
& E(4,9)=-\left(\frac{n}{x_{1}}\right) J_{0}\left(\alpha_{4} x_{1}\right) \\
& E(5, j)=-\left(\frac{n}{x_{1}}\right) J_{0}\left(\alpha_{j}^{1} x_{1}\right) \\
& E(5,5)=-\left[\alpha_{5}^{1} J_{1}\left(\alpha_{5}^{1} x_{1}\right)-\left(\frac{n}{x_{1}}\right) J_{0}\left(\alpha_{5}^{1} x_{1}\right)\right] \\
& E(5, j+5)=\left(\frac{n}{x_{1}}\right) J_{0}\left(\alpha_{j} x_{1}\right) \\
& E(5,9)=-\left[\alpha_{4} J_{1}\left(\alpha_{4} x_{1}\right)-\left(\frac{n}{x_{1}}\right) J_{0}\left(\alpha_{4} x_{1}\right)\right] \\
& E(6, j)=d_{j}^{1} J_{1}\left(\alpha_{j}^{1} x_{1}\right) \\
& E(6, j+5)=-d_{j} J_{1}\left(\alpha_{4} x_{1}\right) \\
& E(7, j)=e_{j}^{1} J_{1}\left(\alpha_{j}^{1} x_{1}\right)
\end{aligned}
$$




$$
\begin{aligned}
& E(8, j)=h_{j}^{1}\left(\frac{n}{x_{1}}\right) J_{0}\left(\alpha_{j}^{1} x_{1}\right)-\left(\alpha_{j}^{1}\right) J_{1}\left(\alpha_{j}^{1} x_{1}\right) \\
& E(9, j+5)=e_{j}\left[-\alpha_{j} J_{1}\left(\alpha_{j} x_{1}\right)+\left(\frac{n}{x_{1}}\right) J_{0}\left(\alpha_{j} x_{1}\right)\right]
\end{aligned}
$$

and the other nonzero elements at the interfaces $\mathrm{x}=\mathrm{x}_{1}$ can be obtained on replacing $\mathrm{J}_{0}$ by $\mathrm{J}_{1}$ and $\mathrm{Y}_{0}$ by $\mathrm{Y}_{1}$ in the above elements. They are $E(i, j+9)(i=1,2,3,4,5,6,9)$. The non - zero elements at $\mathrm{x}=\mathrm{x}_{2}$ by varying $\mathrm{j}$ from 6 to 10 are,

$$
\begin{aligned}
& E(10, j)=\left[\begin{array}{l}
2 \bar{\mu}\left(\frac{\alpha_{j}}{x_{2}}\right) J_{1}\left(\alpha_{j} x_{2}\right) \\
+\left\{-(\bar{\lambda}+2 \bar{\mu})\left(\alpha_{j}\right)^{2}+\bar{\beta} e_{j}-\bar{\lambda} \varepsilon d_{j}+\frac{2 n(n-1) \bar{\mu}}{x_{2}^{2}}\right\} J_{0}\left(\alpha_{j} x_{2}\right)
\end{array}\right] \\
& E(10,9)=-\left[2 n \bar{\mu}\left(\frac{\alpha_{4}}{x_{2}}\right) J_{1}\left(\alpha_{4} x_{2}\right)-\left(\frac{(n-1)}{x_{0}^{2}}\right) J_{0}\left(\alpha_{4} x_{2}\right)\right] \\
& E(10, j+8)=-2 \bar{c}_{66}{ }^{2}\left(\frac{\alpha_{j}^{2}}{x_{2}}\right) J_{1}\left(\alpha_{j}^{2} x_{2}\right)+\left[\bar{c}_{11}\left(\alpha_{j}^{2}\right)^{2}+\bar{c}_{13}^{2} \varepsilon d_{j}^{2}+\bar{e}_{31}^{2} \varepsilon e_{j}^{2}+\bar{\beta} h_{j}\right] J_{0}\left(\alpha_{j}^{2} x_{2}\right) \\
& E(10,18)=2 n \bar{c}_{66}^{2}\left(\frac{\alpha_{4}^{2}}{x_{2}}\right) J_{1}\left(\alpha_{5}^{2} x_{2}\right)-\left[\frac{2 n(n-1) \bar{c}_{66}^{2}}{\left(x_{2}\right)^{2}}\right] J_{0}\left(\alpha_{5}^{2} x_{2}\right) \\
& E(11, j)=2 n \bar{\mu}\left[\left(\frac{\alpha_{j}}{x_{2}}\right) J_{1}\left(\alpha_{j} x_{2}\right)+\left[\frac{(n-1)}{\left(x_{2}\right)^{2}}\right] J_{0}\left(\alpha_{j} x_{2}\right)\right] \\
& E(11,9)=-2 \bar{\mu}\left[\left(\frac{\alpha_{4}}{x_{2}}\right) J_{1}\left(\alpha_{4} x_{2}\right)+\left(\frac{n(n-1)}{\left(x_{2}\right)^{2}}-\left(\frac{\left(\alpha_{4}\right)^{2}}{2}\right)\right) J_{0}\left(\alpha_{4} x_{2}\right)\right] \\
& E(11, j+8)=-2 n \bar{c}_{66}^{2}\left\{\left(\frac{\alpha_{j}^{2}}{x_{2}}\right) J_{1}\left(\alpha_{j}^{2} x_{2}\right)-\left[\frac{(n-1)}{\left(x_{2}\right)^{2}}\right] J_{0}\left(\alpha_{j}^{2} x_{2}\right)\right\} \\
& E(11,18)=-\bar{c}_{66}^{2}\left\{-2\left(\frac{\alpha_{5}^{2}}{x_{2}}\right) J_{1}\left(\alpha_{5}^{2} x_{2}\right)+\left[\left(\alpha_{5}^{2} x_{2}\right)^{2}-\frac{2 n(n-1)}{\left(x_{2}\right)^{2}}\right] J_{0}\left(\alpha_{5}^{2} x_{2}\right)\right\}
\end{aligned}
$$




$$
\begin{aligned}
& E(12, j)=-\bar{\mu}\left(\varepsilon+d_{j}\right)\left[-\alpha_{j} J_{1}\left(\alpha_{j} x_{2}\right)+\left(\frac{n}{x_{2}}\right) J_{0}\left(\alpha_{j} x_{2}\right)\right] \\
& E(12,9)=-\bar{\mu}\left(\frac{n}{x_{2}}\right) \varepsilon J_{0}\left(\alpha_{4} x_{2}\right) \\
& E(12, j+8)=-\left(\varepsilon+d_{j}^{2}+\bar{e}_{15}^{2} e_{j}^{2}\right)\left[\left(\alpha_{j}^{2}\right) J_{1}\left(\alpha_{j}^{2} x_{2}\right)-\left(\frac{n}{x_{2}}\right) J_{0}\left(\alpha_{j}^{2} x_{2}\right)\right] \\
& E(12,18)=\left(\frac{n}{x_{2}}\right) \varepsilon J_{0}\left(\alpha_{5}^{2} x_{2}\right) \\
& E(13, j)=\alpha_{j} J_{1}\left(\alpha_{j} x_{2}\right)-\left(\frac{n}{x_{2}}\right) J_{0}\left(\alpha_{j} x_{2}\right) \\
& E(13,9)=-\left(\frac{n}{x_{2}}\right) J_{0}\left(\alpha_{4} x_{2}\right) \\
& E(13, j+8)=-\alpha_{j}^{2} J_{1}\left(\alpha_{j}^{2} x_{2}\right)+\left(\frac{n}{x_{2}}\right) J_{0}\left(\alpha_{j}^{2} x_{2}\right) \\
& E(13,18)=\left(\frac{n}{x_{2}}\right) J_{0}\left(\alpha_{5}^{2} x_{2}\right) \\
& (14, j)=\left(\frac{n}{x_{2}}\right) J_{0}\left(\alpha_{j} x_{2}\right) \\
& E(14,9)=-\left[\alpha_{4} J_{1}\left(\alpha_{4} x_{2}\right)-\left(\frac{n}{x_{2}}\right) J_{0}\left(\alpha_{4} x_{2}\right)\right] \\
& E(14, j+8)=-\alpha_{j}^{2} J_{1}\left(\alpha_{j}^{2} x_{2}\right)+\left(\frac{n}{x_{2}}\right) J_{0}\left(\alpha_{j}^{2} x_{2}\right) \\
& E(14,18)=\left(\frac{n}{x_{2}}\right) J_{0}\left(\alpha_{5}^{2} x_{2}\right) \\
& E(15, j)=-d_{j} J_{1}\left(\alpha_{4} x_{2}\right) \\
& E(15, j+8)=d_{j}^{2} J_{1}\left(\alpha_{j}^{2} x_{2}\right) \\
& E(16, j+9)=e_{j}^{2} J_{1}\left(\alpha_{j}^{2} x_{2}\right) \\
& E\left(x^{\prime}\right)
\end{aligned}
$$




$$
\begin{aligned}
& E(17, j+9)=h_{j}^{2}\left(\frac{n}{x_{2}}\right) J_{0}\left(\alpha_{j}^{2} x_{2}\right)-\left(\alpha_{j}^{2}\right) J_{1}\left(\alpha_{j}^{2} x_{2}\right) \\
& E(18, j)=e_{j}\left[-\alpha_{j} J_{1}\left(\alpha_{j} x_{2}\right)+\left(\frac{n}{x_{2}}\right) J_{0}\left(\alpha_{j} x_{2}\right)\right]
\end{aligned}
$$

and the other nonzero elements at the interface $x=x_{2}$ can be obtained on replacing $J_{0}$ by $J_{1}$ and $Y_{0}$ by $Y_{1}$ in the above elements. are $E(i, j+4), E(i, j+13)(i=10,11,12,13,14,15), E(16, j+14), E(17, j+14)$ and $E(18, j+4)$ At the outer surface $\mathrm{x}=\mathrm{x}_{3}$, the nonzero elements by varying $\mathrm{j}$ from 14 to 18 are

$$
\begin{aligned}
& E(19, j)=2 \bar{c}_{66}^{2}\left(\frac{\alpha_{j}^{2}}{x_{3}}\right) J_{1}\left(\alpha_{j}^{2} x_{3}\right)-\left[\bar{c}_{11}\left(\alpha_{j}^{2}\right)^{2}+\bar{c}_{13}^{2} \varepsilon d_{j}^{2}+\bar{e}_{31}^{2} \varepsilon e_{j}^{2}+\bar{\beta} h_{j}\right] J_{0}\left(\alpha_{j}^{2} x_{3}\right) \\
& E(19,18)=-2 n \bar{c}_{66}^{2}\left(\frac{\alpha_{4}^{2}}{x_{3}}\right) J_{1}\left(\alpha_{5}^{2} x_{3}\right)+\left[\frac{2 n(n-1) \bar{c}_{66}^{2}}{\left(x_{3}\right)^{2}}\right] J_{0}\left(\alpha_{5}^{2} x_{3}\right) \\
& E(20, j)=\left\{2 n\left(\frac{\alpha_{j}^{2}}{x_{3}}\right) J_{1}\left(\alpha_{j}^{2} x_{3}\right)-\left[\frac{2 n(n-1)}{\left(x_{3}\right)^{2}}\right] J_{0}\left(\alpha_{j}^{2} x_{3}\right)\right\} \\
& E(20,18)=\left\{-2\left(\frac{\alpha_{5}^{2}}{x_{3}}\right) J_{1}\left(\alpha_{5}^{2} x_{3}\right)+\left[\left(\alpha_{5}^{2} x_{3}\right)^{2}-\frac{2 n(n-1)}{\left(x_{3}\right)^{2}}\right] J_{0}\left(\alpha_{5}^{2} x_{3}\right)\right\} \\
& E(21, j)=-\left(\varepsilon+d_{j}^{2}+\bar{e}_{15}^{2} e_{j}^{2}\right)\left[\left(\alpha_{j}^{2}\right) J_{1}\left(\alpha_{j}^{2} x_{3}\right)-\left(\frac{n}{x_{3}}\right) J_{0}\left(\alpha_{j}^{2} x_{3}\right)\right] \\
& E(21,18)=\left(\frac{n}{x_{3}}\right) \varepsilon J_{0}\left(\alpha_{5}^{2} x_{3}\right) \\
& E(22, j)=e_{j}^{2} J_{0}\left(\alpha_{j}^{2} x_{3}\right) \\
& E(22,18)=0 \\
& E(23, j)=h_{j}^{2}\left(\frac{n}{x_{3}}\right) J_{0}\left(\alpha_{j}^{2} x_{3}\right)-\left(\alpha_{j}^{2}\right) J_{1}\left(\alpha_{j}^{2} x_{3}\right) \\
& E(23,18)=0
\end{aligned}
$$


the other nonzero element at the interface $\mathrm{x}=\mathrm{x}_{3}$ can be obtained on replacing $\mathrm{J}_{0}$ by $\mathrm{J}_{1}$ and $\mathrm{Y}_{0}$ by $\mathrm{Y}_{1}$ in the above elements. They are $E(i, j+5)(i=19,20,21,22,23)$. In the case of without voids in the interface region, the frequency equation is obtained by taking $\psi=0$ in Eqn. (14) which reduces to a $21 \times 21$ determinantal equation. The frequency equations derived above are valid for different inner and outer materials of $6 \mathrm{~mm}$ class and arbitrary thickness of layers.

\section{NUMERICAL RESULTS}

Zeros of the frequency equations are evaluated using Muller's method [25]. The elastic, piezoelectric, dielectric and pyroelectric constants for $\mathrm{BaTio}_{3}$ are taken from Ref. [26]-[27]. The material constants of LEMV bonding layer are taken as the hypothetical material no.2, in Table III of Puri and Cowin [28]. The value of dimensionless number $\mathrm{N}$, which is void volume measure factor, defined in eq. (3.4) of Ref [28], and the value of $\mathrm{N}$ is found to be $0 \leq \mathrm{N} \leq 0.66$. The material constants of CFRP bonding layer are taken from [29]. For all the numerical calculations, the inner/outer and middle/outer radius of the cylinders are taken as $\mathrm{a} / \mathrm{c}=0.6666$ and $\mathrm{b} / \mathrm{c}=0.7333$ respectively. The complex frequencies for the flexural waves in the first and second modes are given in Tables 1 and 2. The imaginary parts of the frequencies representing the attenuation of the flexural vibration of Pyro laminated-LEMV (with and without voids) and Pyro laminated - CFRP cylinders are compared with pyroelectric solid cylinder. The dispersion curves for the real part of frequency against the dimensionless wave number for the interfacial layers LEMV ( $\mathrm{N}=0$ and $\mathrm{N}=0.33$ )/CFRP and pyroelectric solid cylinder are plotted for the first and second flexural mode in Figs. 1 and 2 respectively.

\section{CONCLUSION}

The frequency equation for free flexural vibration of pyrocomposite solid cylinder with LEMV as core material is derived. From the numerical data, an increase in imaginary part of the frequencies which is a measure of attenuation of the composite vibration is observed with voids/pores in the core material than the vibration of the pyroelectric solid cylinder. The present model with CFRP core may have a similar practical application discussed in [5]-[6].

\section{REFERENCES}

[1] Lang S. B, "Guide to the literature of piezoelectricity and pyroelectricity, Ferroelectrics" 142, (3-4), 263-379, 1993 and 146, (1-4), 153-369, 1993.

[2] Hasegawa J and Takaya K, "Development of microcomputer- aided pyroelectric thermal imaging system and application to pain management”, Medical and Biological Engg. \& Computing, Vol(24) no(3)/May 1986, 275-280.

[3] Dimmock J O, "Pyroelectric Infrared detectors and applications", Journal of Electronic materials, Vol(1), no(2)/May 1972, 255-309.

[4] Hiroyuki Ida and Jun Kawai, "Identification of steel by X-ray fluorescence analysis with a pyroelectric X-ray generator”, Analytical and Bioanalytical Chemistry, Vol.379, no(4)/2004, 735 -738.

[5] Merabet E K, Yuen H K, Grote W A and Deppermann K L,"A high sensitivity titration calorimeter using pyroelectric sensors”, Journal of Thermal Analysis and Calorimetry, vol. (42), no(5)/Nov,1994

[6] Batra A. K., Simmons M., Padmaia Guggila, Aggarwal M. D., and Lal R. B., "Studies on DTGS: PVDF Composites for Pyroelectric Infrared Detectors”, Integrated Ferroelectric, Vol. 63, 1, 2004, 161-163. 
Table 1 Complex frequencies for different values of real wave numbers in the first flexural mode of the pyrocomposite solid cylinder

\begin{tabular}{|c|c|c|c|c|}
\hline \multirow{3}{*}{$\begin{array}{l}\text { Wave no. } \\
\qquad(\varepsilon)\end{array}$} & \multicolumn{4}{|c|}{ Frequencies } \\
\hline & \multirow{2}{*}{$\begin{array}{c}\text { Pyroelectric solid } \\
\text { cylinder }\end{array}$} & \multicolumn{3}{|c|}{ with core } \\
\hline & & $\begin{array}{c}\text { LEMV } \\
(\mathrm{N}=0)\end{array}$ & LEMV (N=0.33) & CFRP \\
\hline 0.01 & $\begin{array}{c}0.1331 \mathrm{E}+00+\mathrm{i} \\
0.0000 \mathrm{E}+00\end{array}$ & $\begin{array}{c}0.5503 \mathrm{E}-01+\mathrm{i} \\
0.3720 \mathrm{E}-09\end{array}$ & $\begin{array}{c}0.3983 \mathrm{E}-01+\mathrm{i} \\
0.5270 \mathrm{E}-02\end{array}$ & $\begin{array}{c}0.5478 \mathrm{E}-01+\mathrm{i} \\
0.1700 \mathrm{E}-08\end{array}$ \\
\hline 0.2 & $\begin{array}{c}0.8719 \mathrm{E}+00+\mathrm{i} \\
0.0000 \mathrm{E}+00\end{array}$ & $\begin{array}{c}0.7274 \mathrm{E}+00+\mathrm{i} \\
0.2361 \mathrm{E}-07\end{array}$ & $\begin{array}{c}0.7597 \mathrm{E}-01+\mathrm{i} \\
0.1937 \mathrm{E}+00 \\
\end{array}$ & $\begin{array}{c}0.7274 \mathrm{E}+00+\mathrm{i} \\
0.9556 \mathrm{E}-07\end{array}$ \\
\hline 0.6 & $\begin{array}{c}0.1118 \mathrm{E}+01+\mathrm{i} \\
0.0000 \mathrm{E}+00\end{array}$ & $\begin{array}{c}0.9786 \mathrm{E}+00+\mathrm{i} \\
0.1512 \mathrm{E}-11 \\
\end{array}$ & $\begin{array}{c}0.7292 \mathrm{E}+00+\mathrm{i} \\
0.5179 \mathrm{E}+00 \\
\end{array}$ & $\begin{array}{c}0.9786 \mathrm{E}+00+\mathrm{i} \\
0.1468 \mathrm{E}-09 \\
\end{array}$ \\
\hline 1.2 & $\begin{array}{c}0.1455 \mathrm{E}+01+\mathrm{i} \\
0.0000 \mathrm{E}+00 \\
\end{array}$ & $\begin{array}{c}0.1386 \mathrm{E}+01+\mathrm{i} \\
0.8648 \mathrm{E}-08 \\
\end{array}$ & $\begin{array}{c}0.9786 \mathrm{E}+00+\mathrm{i} \\
0.1511 \mathrm{E}-11 \\
\end{array}$ & $\begin{array}{c}0.1311 \mathrm{E}+01+\mathrm{i} \\
0.7133 \mathrm{E}-11 \\
\end{array}$ \\
\hline 1.8 & $\begin{array}{c}0.2193 \mathrm{E}+01+\mathrm{i} \\
0.0000 \mathrm{E}+00\end{array}$ & $\begin{array}{c}0.1985 \mathrm{E}+01+\mathrm{i} \\
0.4822 \mathrm{E}-11\end{array}$ & $\begin{array}{c}0.1752 \mathrm{E}+01+\mathrm{i} \\
0.6017 \mathrm{E}-04\end{array}$ & $\begin{array}{c}0.1808 \mathrm{E}+01+\mathrm{i} \\
0.8440 \mathrm{E}+00\end{array}$ \\
\hline 2.4 & $\begin{array}{c}0.2809 \mathrm{E}+01+\mathrm{i} \\
0.0000 \mathrm{E}+00\end{array}$ & $\begin{array}{c}0.2785 \mathrm{E}+01+\mathrm{i} \\
0.6430 \mathrm{E}-15\end{array}$ & $\begin{array}{c}0.2349 \mathrm{E}+01+\mathrm{i} \\
0.8040 \mathrm{E}-05\end{array}$ & $\begin{array}{c}0.2479 \mathrm{E}+01+\mathrm{i} \\
0.3415 \mathrm{E}-13\end{array}$ \\
\hline 3.0 & $\begin{array}{c}0.3424 \mathrm{E}+01+\mathrm{i} \\
0.0000 \mathrm{E}+00\end{array}$ & $\begin{array}{c}0.3316 \mathrm{E}+01+\mathrm{i} \\
0.1250 \mathrm{E}-12\end{array}$ & $\begin{array}{c}0.2947 \mathrm{E}+01+\mathrm{i} \\
0.3878 \mathrm{E}-05\end{array}$ & $\begin{array}{c}0.3216 \mathrm{E}+01+\mathrm{i} \\
0.2018 \mathrm{E}-07\end{array}$ \\
\hline
\end{tabular}


Table 2 Complex frequencies for different values of real wave numbers in the first flexural mode of the pyrocomposite solid cylinder.

\begin{tabular}{|c|c|c|c|c|}
\hline \multirow{3}{*}{$\begin{array}{l}\text { Wave no. } \\
\qquad(\varepsilon)\end{array}$} & \multicolumn{4}{|c|}{ Frequencies } \\
\hline & \multirow{2}{*}{$\begin{array}{l}\text { Pyroelectric solid } \\
\text { cylinder }\end{array}$} & \multicolumn{3}{|c|}{ Pyrocomposite solid cylinder with core } \\
\hline & & $\begin{array}{l}\text { LEMV } \\
(\mathrm{N}=0)\end{array}$ & $\begin{array}{c}\text { LEMV } \\
(\mathrm{N}=0.33) \\
\end{array}$ & CFRP \\
\hline 0.01 & $\begin{array}{c}0.2563 \mathrm{E}+00+\mathrm{i} \\
0.0000 \mathrm{E}+00\end{array}$ & $\begin{array}{c}0.5813 \mathrm{E}-01+\mathrm{i} \\
0.1626 \mathrm{E}-01\end{array}$ & $\begin{array}{c}0.4830 \mathrm{E}-01+\mathrm{i} \\
0.16115 \mathrm{E}-01\end{array}$ & $\begin{array}{c}0.5503 \mathrm{E}-01+\mathrm{i} \\
0.3720 \mathrm{E}-09\end{array}$ \\
\hline 0.2 & $\begin{array}{c}0.9951 \mathrm{E}+00+\mathrm{i} \\
0.0000 \mathrm{E}+00\end{array}$ & $\begin{array}{c}0.7274 \mathrm{E}+00+\mathrm{i} \\
0.1276 \mathrm{E}-11\end{array}$ & $\begin{array}{c}0.7598 \mathrm{E}-01+\mathrm{i} \\
0.1936 \mathrm{E}-01\end{array}$ & $\begin{array}{c}0.7274 \mathrm{E}+00+\mathrm{i} \\
0.9585 \mathrm{E}-08\end{array}$ \\
\hline 0.6 & $\begin{array}{c}0.1241 \mathrm{E}+00+\mathrm{i} \\
0.0000 \mathrm{E}+00\end{array}$ & $\begin{array}{c}0.1016 \mathrm{E}+01+\mathrm{i} \\
0.4662 \mathrm{E}-08\end{array}$ & $\begin{array}{c}0.7293 \mathrm{E}+00+\mathrm{i} \\
0.5183 \mathrm{E}+00\end{array}$ & $\begin{array}{c}0.1016 \mathrm{E}+01+\mathrm{i} \\
0.2811 \mathrm{E}-07\end{array}$ \\
\hline 1.2 & $\begin{array}{c}0.1577 \mathrm{E}+00+\mathrm{i} \\
0.0000 \mathrm{E}+00\end{array}$ & $\begin{array}{c}0.1386 \mathrm{E}+01+\mathrm{i} \\
0.7573 \mathrm{E}-08\end{array}$ & $\begin{array}{c}0.1016 \mathrm{E}+01+\mathrm{i} \\
0.2811 \mathrm{E}-07\end{array}$ & $\begin{array}{c}0.1311 \mathrm{E}+01+\mathrm{i} \\
0.4283 \mathrm{E}-12\end{array}$ \\
\hline 1.8 & $\begin{array}{c}0.2316 \mathrm{E}+00+\mathrm{i} \\
0.0000 \mathrm{E}+00\end{array}$ & $\begin{array}{c}0.1985 E+01+i \\
0.2282 E-11\end{array}$ & $\begin{array}{c}0.1752 \mathrm{E}+01+\mathrm{i} \\
0.2704 \mathrm{E}-05\end{array}$ & $\begin{array}{c}0.1809 \mathrm{E}+01+\mathrm{i} \\
0.8439 \mathrm{E}+00\end{array}$ \\
\hline 2.4 & $\begin{array}{c}0.2932 \mathrm{E}+01+\mathrm{i} \\
0.0000 \mathrm{E}+00\end{array}$ & $\begin{array}{c}0.2785 \mathrm{E}+01+\mathrm{i} \\
0.5715 \mathrm{E}-15\end{array}$ & $\begin{array}{c}0.2349 \mathrm{E}+01+\mathrm{i} \\
0.1495 \mathrm{E}-06\end{array}$ & $\begin{array}{c}0.2479 \mathrm{E}+01+\mathrm{i} \\
0.5915 \mathrm{E}-13\end{array}$ \\
\hline 3.0 & $\begin{array}{c}0.3548 \mathrm{E}+01+\mathrm{i} \\
0.0000 \mathrm{E}+00\end{array}$ & $\begin{array}{c}0.3316 \mathrm{E}+01+\mathrm{i} \\
0.919 \mathrm{E}-13\end{array}$ & $\begin{array}{c}0.2947 \mathrm{E}+01+\mathrm{i} \\
0.4729 \mathrm{E}-05\end{array}$ & $\begin{array}{c}0.3216 \mathrm{E}+01+\mathrm{i} \\
0.1700 \mathrm{E}-07\end{array}$ \\
\hline
\end{tabular}




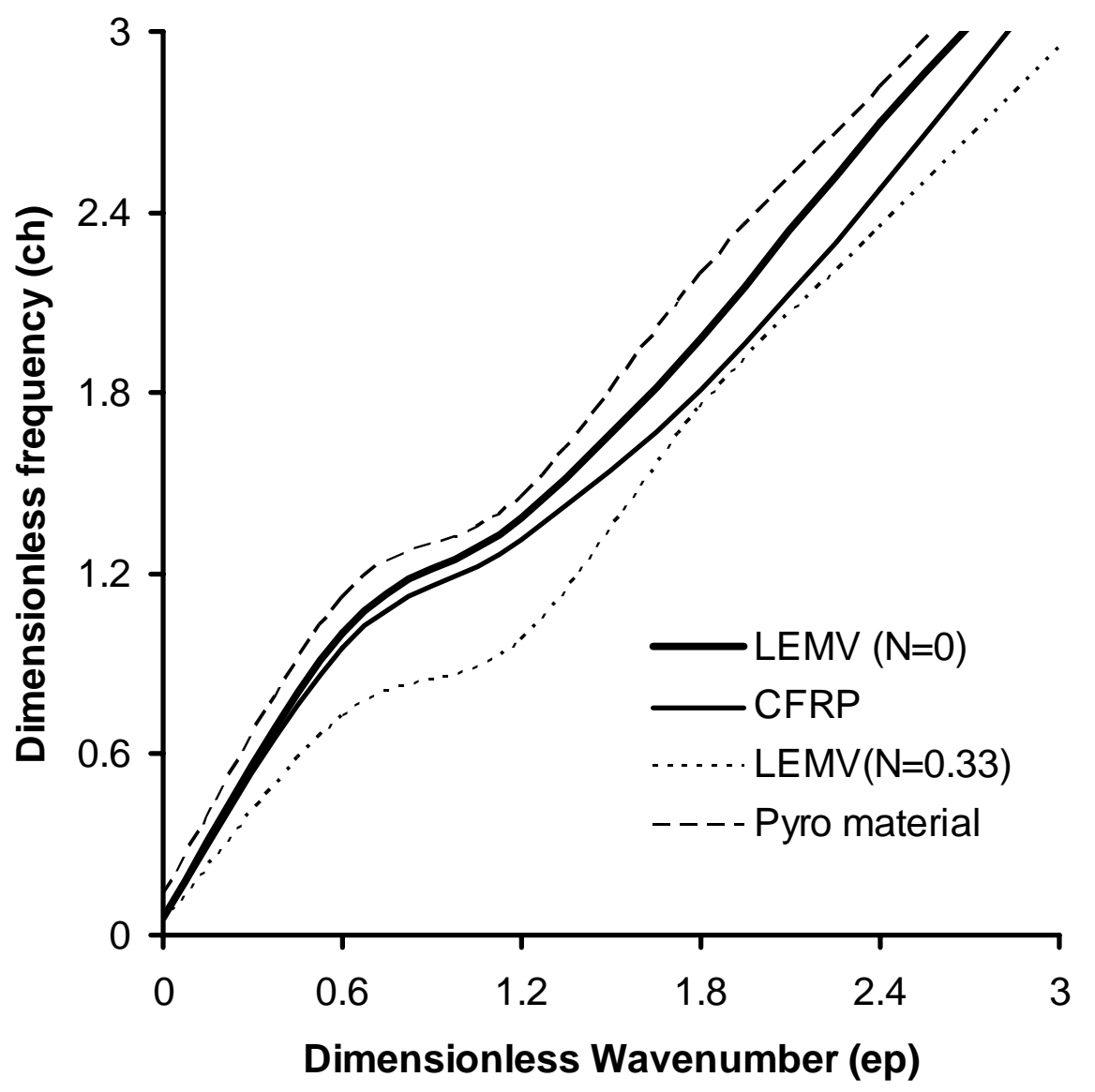

Figure 1 Comparison of dispersion curves of composite solid cylinders $\mathrm{BaTio}_{3} / \mathrm{CFRP} / \mathrm{BaTio}_{3} ; \mathrm{BaTio}_{3} /$ LEMV / $\mathrm{BaTio}_{3}$ (for $\mathrm{N}=0$ and $\mathrm{N}=0.33$ ) and pyroelectric solid cylinder in the first flexural Mode. 


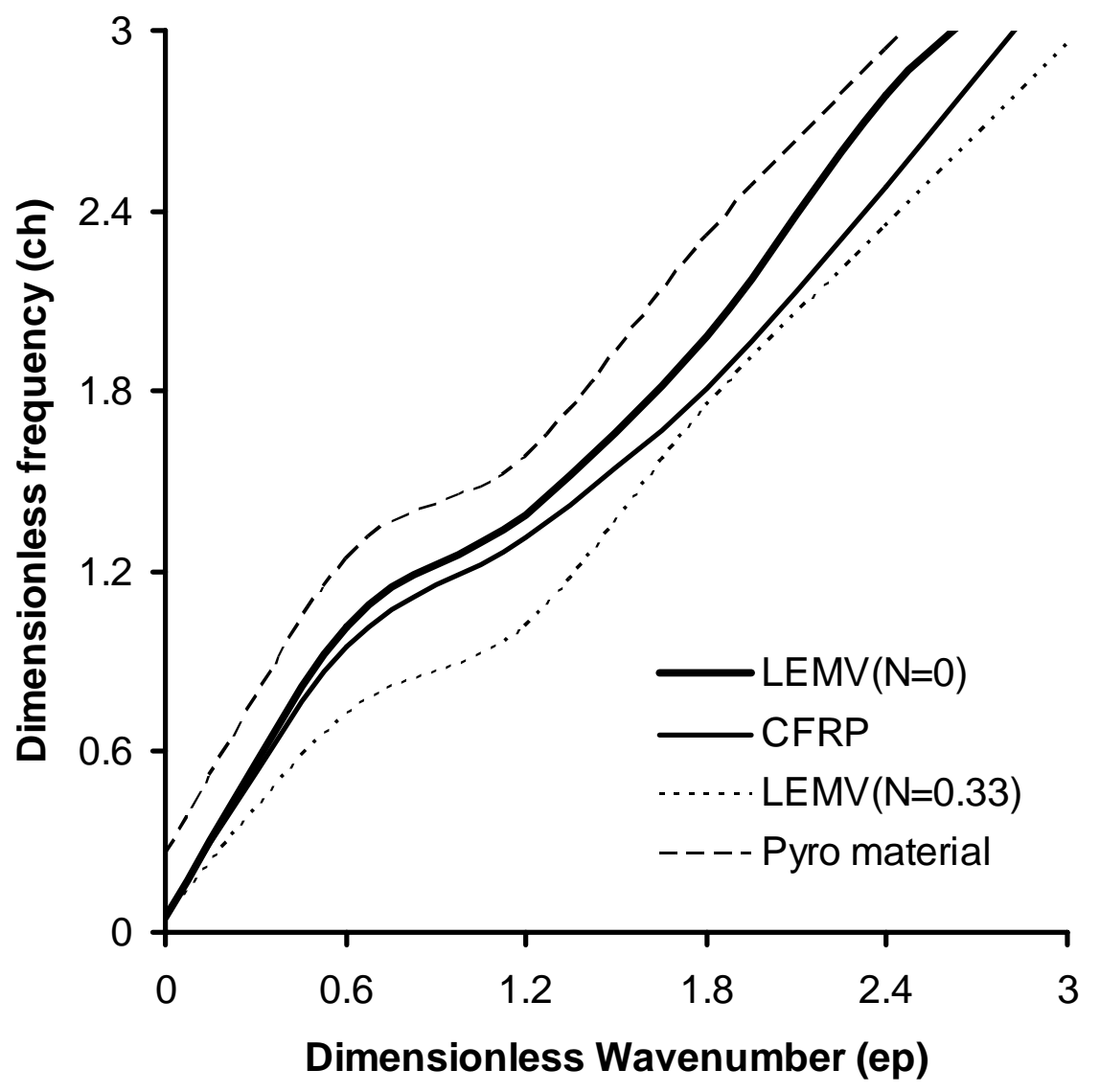

Figure 2 Comparison of dispersion curves of composite solid cylinders $\mathrm{BaTio}_{3} / \mathrm{CFRP} / \mathrm{BaTio}_{3} ; \mathrm{BaTio}_{3} /$ LEMV / $\mathrm{BaTio}_{3}$ (for $\mathrm{N}=0$ and $\mathrm{N}=0.33$ ) and pyroelectric solid cylinder in the second flexural Mode. 
[7] Newnham R. E., Skinner D. P., Klicker K. A., Bhalla A. S., Hardiman B. and Gururaja T. R.,” Ferroelectric Ceramic-Plastic composites for piezoelectric and pyroelectric applications”, Ferroelectrics, Vol. 27, Jan 1980, 49-55.

[8] Dietze M., Krause J., Solterbeck C.,-H., and Es-Souni M.,” Thick film polymer-ceramic composites for pyroelectric applications”, J.Appl. Phy., Vol.101, 5, 2007, 054113-054113-7.

[9] Ploss B., Wong Y. W., and Shin F. G., "Pyroelectric Ceramic/Polymer Composite with Electrically Conducting Matrix Material”, Ferroelectrics, Vol. 325, Sep. 2005, 165-169.

[10] Ploss B.,” Improving the pyroelectric Coefficient of Ceramic/Polymer Composite by Doping the Polymer Matrix”, Ferroelectrics, Vol. 338, August 2006, 145-151.

[11] Paul H S and Raman G V, “Vibrations of Pyroelectric plates”, J. Acoust. Soc. Am, 90 (4), 1991.

[12] Paul. H. S and Raman. G. V, "Wave propagation in a hollow Pyroelectric circular cylinder of crystal class 6”, Acta Mechanica, 87, 1991, 37-46.

[13] Paul. H. S and Raman. G. V, "Wave propagation in a pyroelectric Cylinder of arbitrary cross section with a circular cylindrical cavity”, J. Acoust. Soc. Am, 93, 1993, 1175.

[14] Paul H. S. and Nelson V. K., "Flexural Vibration of Piezocomposite Hollow Circular cylinder", J.Acoust.Soc.Am., Vol.99, 1996, 309-313.

[15] Vasudeva. R. Y. and Govinda Rao. P, "Influence of voids in interface zones on lamb wave propagation in composite plates",

J. Acoust.soc.Am., 89, 1991, 516 - 522.

[16] Vasudeva. RY and Govinda Rao. P, "Influence of voids in interface zones on lamb mode spectra in fiber - reinforced composite laminates”,J. Appl. Phys 71,1991, 612 - 619.

[17] Cowin S. C. and Nunziato J. W.,’Linear Elastic Materials with voids”, J. Elasticity, 13, 1983, 125-147.

[18] Harper B D, Staab G H and Chen R C, "A Note on the Effects of Voids Upon the Hygral and Mechanical properties of AS4/3502 Graphite/Epoxy”, J. composite material, 21, 1987, 280 - 289.

[19] Voorhees E J V and Green D J, “Mechanical Behavior of a Ceramic Sandwich Composite System”, J. composite materials, 26, 1992, $1664-1677$.

[20] Takagikiyoshi, Sato Hiroshi, Saigo Muneharu, and Smith Ralph

C., modeling, signal processing, and control. Conference, San Diego CA, ETATS-UNIS, Vol.5757, 2005, $471-280$.

[21] Mindlin. R. D,” On the equations of motion of piezoelectric crystals”, Problems in Continuum Mechanics, SIAM, , 1961, 282 - 290.

[22] Mindlin. R. D, "Equations of High Frequency vibrations of Thermo Piezoelectric crystal plate”, Int. J. Solids structures, Vol.10, 1974, PP 625-637.

[23] Cowin S. C. and Puri P.,” The classical pressure vessel problems for linear elastic materials with voids”, J. Elasticity, 13, 1983, 157- 163.

[24] Atkin. R. J, Cowin. S. C and Fox. N, “On boundary conditions for polar materials”, zeitschrift für angewandte Mathematik und phhysik, 28, 1977, 1017.

[25] Jain M. K., Iyengar S. R. K. and Jain R. K., "Numerical Methods for Scientific and Engineering Computation”, Wiley, New York, $2^{\text {nd }}$ ed, 1987.

[26] Berlincourt. D. A., Curran.D.R, Jaffe. H, “Physical acoustics”, Vol.1, Pt.A, pp.204 - 206, New York, Academic 1964.

[27] Condan. E. V., Odishaw. H, “American institute of physics handbook”, pp.9-118. New York, McGraw Hill 1972.

[28] Puri P. and Cowin S. C., "Plane waves in linear elastic materials with voids”, J. Elasticity, 15, 1985, 167 $-183$.

[29] Ashby. M. F and Jones. D. R. H, “Engineering Materials 2”, Pergamon Press, 1986. 